\title{
The Performance of Rectangular Microstrip Structure by Applying the Cole-Cole Diagram Concept
}

\author{
Settapong Malisuwan, Jesada Sivaraks, and Nattakit Suriyakrai
}

\begin{abstract}
This research provides a method for analyzing the performance of a rectangular microstrip structure by adopting Cole-Cole diagram. A "reactive relaxation" concept is introduced to represent the frequency-dependent characteristics of a microstrip. Included in the algorithm are relevant considerations relevant to the substrate dielectric and strip-line conductor losses. The proposed concept is used to analyze the microstrip structure performance. The proposed models are set up for use in computer-aided microstrip design and are fully compatible with the needs and trends of modern computer-aided microstrip antenna design and RF integrated circuit (RFID) design.
\end{abstract}

Index Terms-Dielectric substrate, cole-cole diagram, frequency-dependent, microstrip structure.

\section{INTRODUCTION}

Microstrip structure is one of the most popular types of planar transmission lines, primarily because it is easily integrated with other passive and active microwave devices. Relevant design equations in closed-form using semi-empirical strategies specifying the frequency-dependent, effective dielectric permittivity concept and dispersion characteristics of a microstrip structure have been derived in the existing literature as elaborated in [1], [2]. While most computer-aided design (CAD) are developed using algorithms with built-in microstrip design capabilities, simple calculation methods for microstrip structure parameters by hand-calculator and/or by personal computer is preferable for for preliminary design purposes, and/or for quick circuit evaluation purposes. However, it is required that the physical considerations of microstip circuits be considered on a step-by-step basis. Therefore, many researchers are in search of simple methods, which are at the same time and sufficient to explain the physical aspects of microstrip circuits, precisely.

The main contribution of this paper is to analyze the microstrip structure performance based on the Cole-Cole diagram representation [3]. To achieve this goal, an approach that uses the Debye relation [3] is introduced to portray such frequency-dependent characterization of a microstrip structure. Hence, "a reactive relaxation diagram" (analogous to the Cole-Cole diagram) is proposed to represent the frequency-dependent capacitive effects as well as the associated loss in a microstrip structure. The proposed model

Manuscript received July 3, 2013; revised September 3, 2013. Full financial support for this paper is from National Broadcasting and Telecommunications Commission.

The authors are with the National Broadcasting and Telecommunications Commission, Bangkok, Thailand (e-mail: settapong.m@nbtc.go.th, jesada.s@nbtc.go.th, nattakit.s@nbtc.go.th). is conducive for computer-aided microstrip circuit designs. There is a gap in the research as the Cole-Cole model proposed with respect to microstrip structure application as a microstrip patch antenna has not been reported elsewhere.

\section{General Structure of Microstrip PATCH Antenna}

A microstrip antenna consist of a dielectric substrate which is in between the radiating patch which is on top and the ground plane which is on the other side as illustrated in in Fig. 1 . The patch is usually made of gold or copper (conducting material) and the shape varies. The radiating patch and the feed lines are usually photo etched on the dielectric substrate.

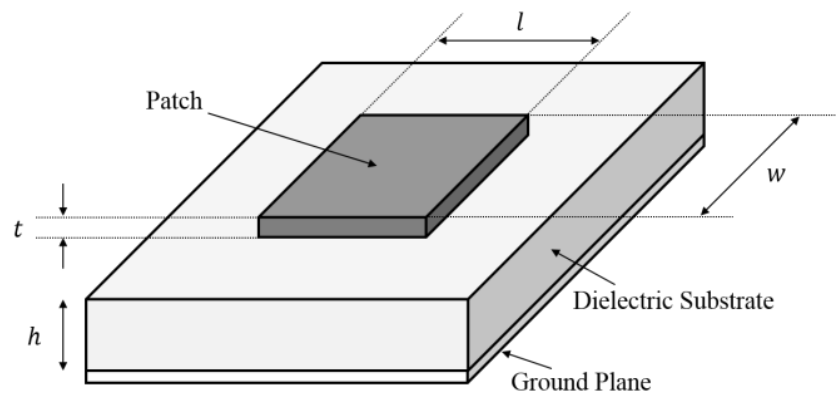

Fig. 1. Structure of a microstrip patch antenna.

To decrease the complexity of the analysis, the patch is generally square, rectangular, circular, triangular and elliptical or some other common shape. For a rectangular patch, the length $\mathrm{L}$ of the patch is usually in the range of $0.3333 \lambda_{o}<L<0.5 \lambda_{o}$, where $\lambda_{o}$ is the free space wavelength. The patch is proposed is very thin such that $t<<\lambda_{o}$ (where $t$ is the patch thickness). The height $h$ of the substrate is usually $0.003 \lambda_{o} \leq h \leq 0.05 \lambda_{o}$. The dielectric constant of the substrate $\epsilon r$ is typically in the range $2.2 \leq$ $\epsilon_{r} \leq 12$ [4].

Normally, it would be very time consuming and expensive to employ numerical approaches, requiring high performance computer, for microstrip synthesis (or analysis) leading to computer-aided circuit design. On the contrary, the expressions derived in this chapter are simpler and computationally economical in terms of time and system requirements.

The design formulas derived first refer to the characteristic impedance and effective dielectric permittivity of microstrip structure, the depict approximations to rigorous quasi-static solutions [5], [6]. Then, the dielectric relaxation concept is described to fuse microstrip design formulations into it.

\section{A. The Characteristic Impedance}

This model refers to the impedance of the microstrip structure in a homogeneous medium. The substrate material 
and the material above the conductor are assumed to be the same. Consider the case where the substrate permittivity is air $\left(\epsilon_{r}=1\right)$; that is, the line is floating on the ground plane. This impedance expression is rewritten here [5]:

$$
Z_{0}(0)=\frac{\eta_{0}}{2 \pi} \ln \left(\frac{f(u)}{u}+\sqrt{1+\left(\frac{2}{u}\right)^{2}}\right)
$$

where

$$
f(u)=6+(2 \pi-6) \exp \left[-\left(\frac{30.666}{(u)}\right)^{0.7528}\right]
$$

and $\eta_{0}$ is the wave or intrinsic impedance of the free space $(377 \Omega)$. The subscript 0 in Eqn. (1) indicates dielectric of air and the argument zero refers to static values.

\section{B. The Effective Permittivity}

For a structure shown in Fig. 1, the effective permittivity is rewritten here as [5]

$$
\varepsilon_{e f f}(0)=\frac{\varepsilon_{r}+1}{2}+\frac{\varepsilon_{r}-1}{2} q
$$

where $q$ is a filling-factor which can be found in [5].

\section{Frequency-Dependent Effective Permittivity}

The effective permittivity of the microstrip structure would change with frequency due to dispersion effects. The effective permittivity increases with frequency and asymptotically approaches $\varepsilon_{r}$. The due to dispersion the effects become significant particularly for high frequencies (above $4 \mathrm{GHz}$ ).

The frequency-dependence of the effective dielectric constant is specified by the following empirical relation [7]:

$$
\varepsilon_{e f f}(\omega)=\varepsilon_{r}+\frac{\varepsilon_{e f f}(0)-\varepsilon_{r}}{1+Q(\omega)}
$$

where $Q(\omega)$ can be found in[7] and $\omega_{n}^{*}=2 \pi f_{n}^{*}\left(f_{n}^{*}\right.$ in GHz.mm) is the frequency ratio. That is,

$$
f_{n}^{*}=k\left(\frac{f}{f_{0}}\right)\left(\frac{h}{\lambda_{0}}\right)=k\left(\frac{l}{\lambda_{0}}\right)\left(\frac{h / \lambda_{0}}{l / \lambda_{0}}\right)
$$

where $k=300$ and $l$ is the line-length.

\section{GEOMETRICAL REPRESENTATION OF DiELECTRIC RELAXATION PROCESS}

\section{A. Dielectric Permittivity}

The dielectric permittivity $(\varepsilon)$ of a material can be defined as the ratio of the electric field $E_{0}$ in free-space to that is, material $E$ for the same distribution of charge. This quantity is attributed as a measure in capacitors, the most simple of which consists of parallel plates of area $A$ separated by a distance $d$, which is very small in comparison with $A$.

If the charges on the plates remain unchanged and the space between them is filled with a dielectric material, the capacitance $C$ of the condenser is

$$
C=A \varepsilon / d
$$

where $\varepsilon=\varepsilon_{0} \varepsilon_{r}$ and $\varepsilon_{0}=(1 / 36 \pi) \times 10^{-9} \mathrm{~F} / \mathrm{m}$ denotes the free-space permittivity.

\section{B. Dielectric Relaxation Process}

In general, the term relaxation means the time-dependent return to equilibrium of a system that has been perturbed by a change in an applied constraint. When the constraint is the electric field as in a capacitor, the observable quantity that varies with time is the dielectric polarization, giving rise to a dielectric relaxation.

The relaxation processes are very important for the interactions between fields and dielectrics. The Debye model describes the materials in terms of individual dipole moments containing variable charge separation in a viscous medium. A dipole subjected to an electric field would tend to align itself and elongate in the direction of the applied electric field in a finite time. If the externally applied field is abruptly cut off, all the torques due to the external field on the dipoles vanish instantly, and, the dipole "relaxes" to the initial state as an exponential decay process.

The application of a constant electric field $E$ to a dielectric resolves into the development of an electric displacement $D$ that increases with time until a constant value is reached if time is sufficiently long. After removal of the electric field, the electric displacement returns with time to zero.

At sufficiently small electric displacements, $\varepsilon=|D| /|E|$; that is, the system is linear, and, consequently, it conforms to the superposition principle [3]. The dielectric constant is a time-dependent property, its value creasing from $\varepsilon_{\infty}$ for $t=0$ to $\varepsilon_{s}$ for $t \rightarrow \infty$. The electric displacement can obviously be written as

$$
D(t)=\left[\varepsilon_{\infty}+\left(\varepsilon_{s}-\varepsilon_{\infty}\right) \Phi(t)\right] E
$$

where $\Phi(t)$ is the built-up normalized dielectric function whose extreme values are

$$
\begin{array}{cc}
\Phi(t)=1-\phi(t)=0 & \text { for } t=0 \\
\Phi(t)=1-\phi(t)=1 & \text { for } t=\infty
\end{array}
$$

where $\phi(t)$, the normalized decay function of the polarization when a steady macroscopic electric field is removed from the medium, acquires the values 1 and 0 at $t=0$ and $t=\infty$, respectively. If an electric field is applied at $t=0$, but it increases by an infinitesimal amount, $d E$, at $t=\theta(0<\theta<t)$, the increase in electric displacement at $t$ will be

$$
d D=\varepsilon_{\infty} d E+\left(\varepsilon_{s}-\varepsilon_{\infty}\right) \Phi(t=\theta) d E
$$

In linear system, the total displacement at time $t$ caused by a variable electric field $E(\theta)$ is the result of the superposition of all the increments, $\mathrm{dD}$ and

$$
D(t)=\varepsilon_{\infty} E(t)+\left(\varepsilon_{s}-\varepsilon_{\infty}\right) \int_{-\infty}^{t} \frac{d E(\theta)}{d \theta} \Phi(t-\theta) d \theta
$$

By making the substitution $(t-\theta)=\xi$ and integrating by parts, one finds 


$$
D(t)=\varepsilon_{\infty} E(t)+\left(\varepsilon_{s}-\varepsilon_{\infty}\right) \int_{0}^{\infty} \frac{d \Phi(\xi)}{d \xi} E(t-\xi) d \xi
$$

For an alternating electric field, $E=E_{0} \exp (j \omega t)$, Eqn. (11) leads to

$$
\begin{gathered}
\varepsilon_{m}^{*}(\omega)=D(t) / E(t)= \\
\varepsilon_{\infty}+\left(\varepsilon_{s}-\varepsilon_{\infty}\right) \int_{0}^{\infty} \exp (-j \omega \xi)\left[-\frac{d \phi(\xi)}{d \xi}\right] d \xi
\end{gathered}
$$

where $-d \phi(\xi) / d \xi=d \phi(\xi) / d \xi$, and $\varepsilon_{m}^{*}(\omega)=\varepsilon_{m}^{\prime}(\omega)-$ $j \varepsilon_{m}^{\prime \prime}(\omega)$ is the complex dielectric permittivity. Rearrangement of the terms of Eqn. (12) gives [3]

$$
\frac{\varepsilon_{m}^{*}(\omega)-\varepsilon_{\infty}}{\varepsilon_{s}-\varepsilon_{\infty}}=\int_{0}^{\infty}(-d \phi / d t) \exp (-j \omega t) d t
$$

Solution of the integral requires knowing the decay function over the entire relaxation range. In an ideal relaxation range, the rate of return to the equilibrium, $\mathrm{P}_{\mathrm{eq}}$, and, as a first approximation, the phenomenon may be described by a linear first-order approximation with the solution $P-P_{e q} \approx \exp \left(-t / \tau_{r}\right)$. Parameter $\tau_{\mathrm{r}}$ is called the relaxation time, which may be defined as the time in which the observable quantity is reduced to $1 / e$ times its original value. For a dielectric relaxation involving a single relaxation time (Debye relaxation), $\phi(t) \approx \exp \left(-t / \tau_{r}\right)$ and Eqn. (13) becomes

$$
\begin{gathered}
\frac{\varepsilon_{m}^{*}(\omega)-\varepsilon_{\infty}}{\varepsilon_{s}-\varepsilon_{\infty}}=\frac{1}{1+j \omega \tau_{r}} \\
\varepsilon_{m}^{*}(\omega)=\varepsilon_{\infty}+\frac{\varepsilon_{S}-\varepsilon_{\infty}}{1+j 2 \pi\left(\omega / \omega_{r}\right)}
\end{gathered}
$$

where $\varepsilon_{m}^{*}(\omega)$ is the complex permittivity of the dielectric material; $\varepsilon_{\infty}$ is the permittivity at infinite frequency; $\varepsilon_{s}$ is the static or d.c. permittivity; $\omega=2 \pi f ; f$ is the applied frequency; and $\tau_{r}\left(1 / f_{r}\right)$ is the relaxation time of the dielectric material and it can be decomposed as:

$$
\begin{gathered}
\varepsilon_{m}^{*}(\omega)= \\
{\left[\varepsilon_{\infty}+\frac{\varepsilon_{s}-\varepsilon_{\infty}}{1+4 \pi^{2}\left(\omega / \omega_{r}\right)^{2}}\right]-j\left[\left(\varepsilon_{s}-\varepsilon_{\infty}\right) \frac{2 \pi\left(\omega / \omega_{r}\right)}{1+4 \pi^{2}\left(\omega / \omega_{r}\right)^{2}}\right]}
\end{gathered}
$$

Therefore, the real $\varepsilon_{m}^{\prime}(\omega)$ and loss $\varepsilon_{m}^{\prime \prime}(\omega)$ components of $\varepsilon_{m}^{*}(\omega)$ are given by

$$
\begin{gathered}
\varepsilon_{m}^{\prime}(\omega)=\left[\varepsilon_{\infty}+\frac{\varepsilon_{S}-\varepsilon_{\infty}}{1+4 \pi^{2}\left(\omega / \omega_{r}\right)^{2}}\right] \\
\varepsilon_{m}^{\prime \prime}(\omega)=\left[\left(\varepsilon_{s}-\varepsilon_{\infty}\right) \frac{2 \pi\left(\omega / \omega_{r}\right)}{1+4 \pi^{2}\left(\omega / \omega_{r}\right)^{2}}\right]
\end{gathered}
$$

Eqn. (17) and (18) are usually called Debye equations. The functions represented by Eqn. (17) and (18) are illustrated in Fig. 2.

Experimentally, one measures usually the quantities $\varepsilon_{m}^{\prime}(\omega)$ and the loss tangent [8]:

$$
\tan \left(\delta_{d}\right)=\frac{\varepsilon^{\prime \prime}(\omega)}{\varepsilon^{\prime}(\omega)}
$$

and it is convenient to know the Debye equations in terms of the loss tangent, when

$$
\tan \left(\delta_{d}\right)=\frac{\left(\varepsilon_{S}-\varepsilon_{\infty}\right) \omega \tau_{r}}{\varepsilon_{S}+\varepsilon_{\infty} \omega^{2} \tau_{r}^{2}}
$$

In the limit of low and high frequencies, Eqn. (17) and (18) lead to

$$
\begin{aligned}
\lim _{\omega \rightarrow 0} \varepsilon_{m}^{\prime}(\omega) & =\varepsilon_{s} \\
\lim _{\omega \rightarrow \infty} \varepsilon_{m}^{\prime}(\omega) & =\varepsilon_{\infty}
\end{aligned}
$$

A close examination of Eqn. (17) and (18) suggests that whereas $\varepsilon^{\prime}(\omega)$ is a continuously decreasing function of frequency, $\varepsilon^{\prime \prime}(\omega)$ approaches zero both for small and for large values of frequency, reaching a maximum at $\omega \tau_{\mathrm{r}}=1$. That is, the relaxation time is equal to the reciprocal of the angular frequency at the maximum of the loss absorption. The simple Debye relation is derived on the following assumptions:

1) Local field at any point in the medium is the same as the applied field.

2) D.C. conductivity of the material is negligible.

3) All the dipoles have identical relaxation time $\tau_{r}$.

The real and imaginary parts of the Debye relation are depicted in Fig. 2. The Debye relation can be represented as shown in Fig. 3. It is known as the Cole-Cole diagrams. This graphical representation which is of considerable practical importance involves plotting $\varepsilon^{\prime}$ versus $\varepsilon^{\prime \prime}$. The function $\varepsilon^{\prime \prime}\left(\varepsilon^{\prime}\right)$ can be obtained by elimination of $\omega$ between the equations $\varepsilon_{m}^{\prime}(\omega)$ and $\varepsilon_{m}^{\prime \prime}(\omega)$. The functional relation obtained in this format is depicted as a circle. $\varepsilon^{*}$ is now presented by the semi-circle of radius $\left(\varepsilon_{s}-\varepsilon_{\infty}\right) / 2$ centered at $\varepsilon^{\prime}=\left(\varepsilon_{\infty}+\varepsilon_{s}\right) / 2$. The top of this semi-circle corresponds to $\omega \tau_{r}=1$. The relaxation time is calculated from $\tau_{r}=1 / f_{r}$. It can be seen that $\varepsilon^{\prime}$ decreases with frequency. This means that the energy $\varepsilon^{\prime}|E|^{2} / 2$ stored in the material will decrease with frequency.

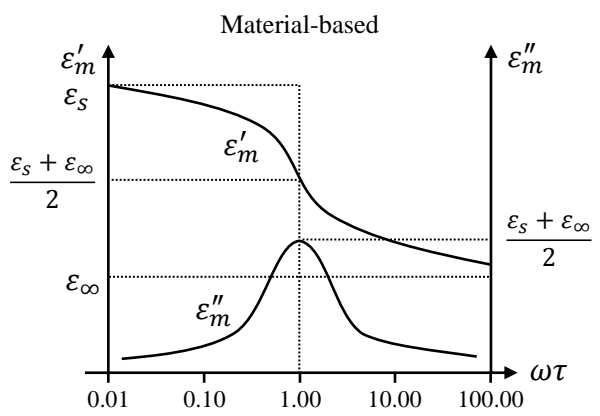

Fig. 2. Dielectric relaxation: debye relation.

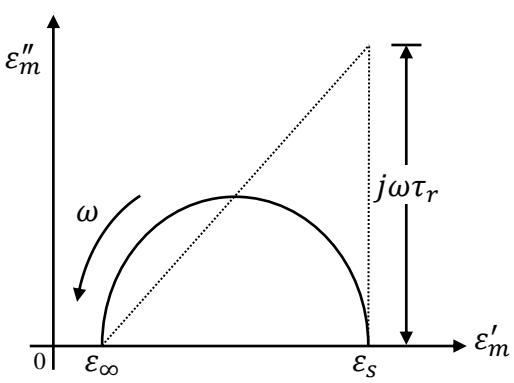

Fig. 3. Cole-cole diagram of material-based. 


\section{MicRostrip-BASED EQuivalent RELAXATION PROCESS}

The concept of dielectric relaxation can be used to characterize the frequency-dependent microstrip structure performance. For this purpose the real part of the Debye relation can be equated to the equivalent frequency-dependent permittivity (Eqn. (4)) deduced for a microstrip. That is,

$$
\varepsilon_{u}^{\prime}(\omega)=\varepsilon_{e f f}(\omega)=\left[\varepsilon_{r}+\frac{\varepsilon_{e f f}(0)-\varepsilon_{r}}{1+Q(\omega)}\right]
$$

From Kirschning and Jansen' frequency-dependent effective permittivity in Eqn. (22), it can be equated to Eqn. (17) as follows.

$$
\varepsilon_{r}+\frac{\varepsilon_{e f f}(0)-\varepsilon_{r}}{1+Q(\omega)}=\varepsilon_{\infty}+\frac{\varepsilon_{s}-\varepsilon_{\infty}}{1+4 \pi^{2}\left(\omega / \omega_{r}\right)^{2}}
$$

This gives,

$$
\begin{gathered}
\varepsilon_{r}=\varepsilon_{\infty} \\
\varepsilon_{e f f}(0)=\varepsilon_{s} \\
\tau_{0}=\frac{\sqrt{Q(\omega)}}{\omega}
\end{gathered}
$$

Now, an "imaginary part" of the equivalent permittivity of a microstrip system can be obtained by applying the Equations (24)-(26) into the imaginary part of Equation. (16) $\left(\varepsilon_{m}^{\prime \prime}(\omega)\right)$. Hence, the imaginary part of Cole-Cole expression for a microstrip system can be written as

$$
\varepsilon_{u}^{\prime \prime}(\omega)=\left[\frac{\left(\varepsilon_{e f f}(0)-\varepsilon_{r}\right) \sqrt{Q(\omega)}}{1+Q(\omega)}\right]
$$

Hence, the complex permittivity of microstrip system in compact form can be written as:

$$
\varepsilon_{u}^{\prime \prime}(\omega)=\varepsilon_{r}+\frac{\varepsilon_{e f f}(0)-\varepsilon_{r}}{1+j(1 / 2 \pi)\left(\omega_{0} / \omega\right)}
$$

\section{MATERIAL-BASED VERSUS MicROSTRIP-BASED COLE-COLE DiagRam}

The difference between the material-based Debye relation and that of microstrip-based considerations is considered in this subsection. By illustrating the relevant Debye relations via Cole-Cole diagrams, a lateral inversion as shown in Eqn. (15) and (28) can be noticed. That is, the material-based and microstrip-based Cole-Cole diagrams are laterally inverted in a mirror-image fashion. Further, the maximum points of semi-circles in the Cole-Cole patterns correspond to maximum Debye loss in a lossy dielectric material; but, in respect of a microstrip system, these points can be regarded as to depict the maximum reactive (capacitive) energy confined within the microstrip structure. That is, pertinent to these maximum value points (A) in Fig. 4(a), it can be considered that the microstrip geometry holds the field within itself, rather than letting it fringe out. In material-based diagram (Fig. 5(a)), it can be observed that loss in the material increases with dielectric constant. In the microstrip-based diagram (Fig. 4(a)), there is a reduction in the extent of energy confined when $u=(w / \lambda)(h / \lambda)$ ratio is increased. That is because, an increase in fringing field results in with decreasing substrate thickness.

TABLE I: MATERIAL-BASED VERSUS MICROSTRIP-BASED COLE-COLE DIAGRAM

\begin{tabular}{|l|l|}
\hline \multicolumn{1}{|c|}{ Material-based } & \multicolumn{1}{c|}{ Microstrip-based } \\
\hline $\begin{array}{l}\varepsilon_{m}^{\prime \prime}(\omega) \text { represent the lossy } \\
\text { parameter of the dielectric } \\
\text { material. }\end{array}$ & $\begin{array}{l}\varepsilon_{u}^{\prime \prime}(\omega) \text { represent the confined energy } \\
\text { within the microstrip. }\end{array}$ \\
\hline $\begin{array}{l}\text { Relaxation time }\left(\tau_{r}=1 / f_{r}\right) \\
\text { - The time constant for } \\
\text { exponential decay to the initial } \\
\text { state after the externally applied } \\
\text { field is abruptly cut off in the } \\
\text { material. }\end{array}$ & $\begin{array}{l}\text { "Reactive relaxation time }\left(\tau_{0}=\right. \\
1 f \sigma^{\prime} \\
\text { - The time constant needed to store the } \\
\text { energy }\left(\varepsilon_{u}^{\prime \prime}\left(f_{0}\right)\right) \text { due to abruptly } \\
\text { applied electric field in the microstrip } \\
\text { circuit. }\end{array}$ \\
\hline $\begin{array}{l}\varepsilon_{\infty}=\text { Dielectric constant at } \\
\text { infinite frequency }\end{array}$ & $\begin{array}{l}\varepsilon_{e f f}(0)=\text { Static effective permittivity } \\
\text { dimension-dependent of the } \\
\text { microstrip structure }\end{array}$ \\
\hline $\begin{array}{l}\varepsilon_{s}=\text { Static (d.c.) dielectric } \\
\text { constant }\end{array}$ & $\begin{array}{l}\varepsilon_{r}=\text { Dielectric constant of the } \\
\text { substrate }\end{array}$ \\
\hline
\end{tabular}
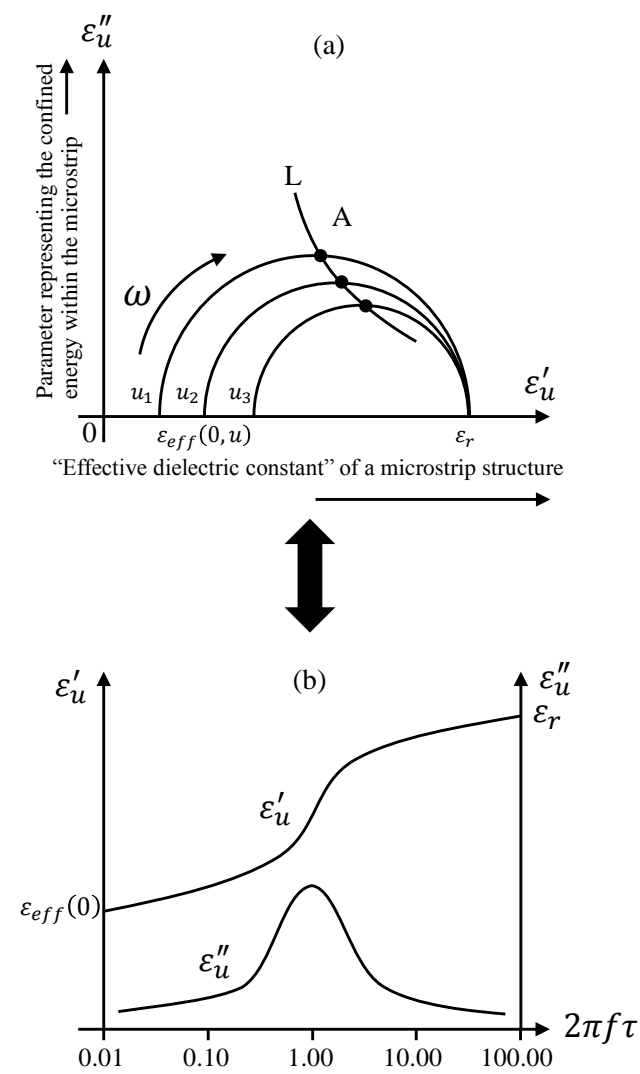

Fig. 4 (a) Cole-Cole representation of reactive relaxation of a microstripstructure with $u=(w / \lambda)(h / \lambda)$ and $u 1<u 2<u 3$. (b) Equivalent Debye relation: microstrip-based. 

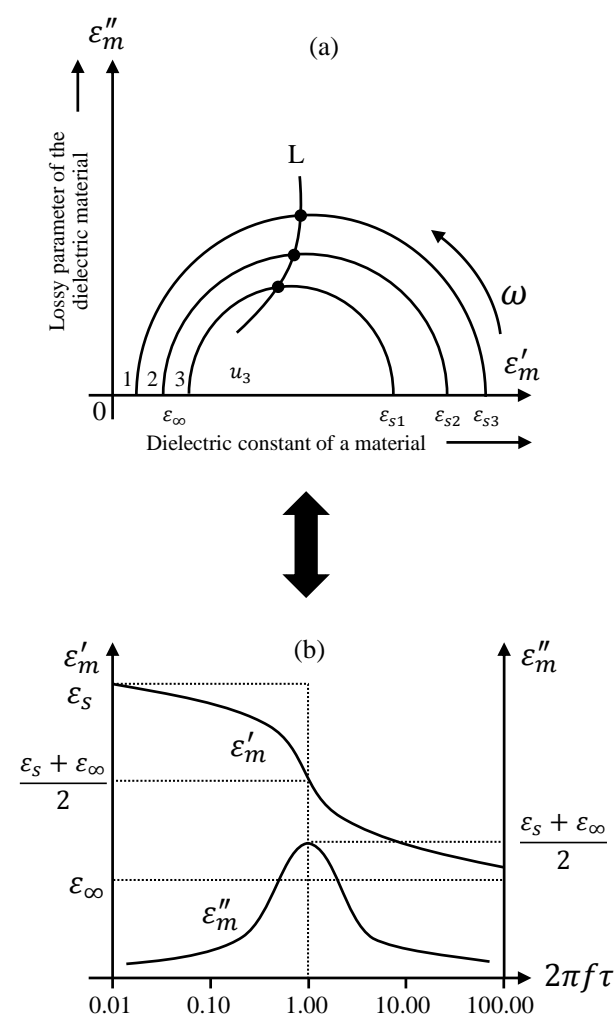

Fig. 5 (a) Cole-Cole diagram of Debye relaxation in a dielectric material with $\varepsilon_{\mathrm{s} 1}<\varepsilon_{\mathrm{s} 2}<\varepsilon_{\mathrm{s} 3}$ and $\tau_{r}\left(\varepsilon_{\mathrm{s} 1}\right)<\tau_{r}\left(\varepsilon_{\mathrm{s} 2}\right)<\tau_{r}\left(\varepsilon_{\mathrm{s} 3}\right)$. (b) Debye relation: material-based.

\section{EFFECTS OF LOSSY FACTORS}

The equivalent Cole-Cole diagram for the microstrip structure (Fig. 4) can be further modified to include the lossy attributions. That is, in the event that the stripline has a lossy substrate and/or when the metallic strip poses a conduction loss, the relevant considerations can be appropriately included in the Cole-Cole representation of Fig. 4.

The conductor loss of the strip can be added as a lossy component into the imaginary part of the equivalent permittivity of the microstrip system. Considering the attenuation (in $\mathrm{Np} / \mathrm{m}$ ) due to the transmission along a microstrip conductor as specified in [5]:

$$
a_{c}(f)=\frac{\sqrt{\varepsilon_{e f f}(0, u)} \exp \left[-1.2\left(Z_{0}(0) / \eta_{0}\right)^{0.7}\right] \sqrt{\pi f \mu_{0}}}{Z_{0}(0) w \sqrt{\sigma_{c}}}
$$

This conductor-loss can be added as a lossy component into the imaginary part of the equivalent complex permittivity of the microstrip system. The attenuation (in $\mathrm{Np} / \mathrm{m}$ ) due to the transmission along the microstrip conductor can be specified as:

$$
a_{c} \cong \sqrt{\pi f \mu_{0} \sigma_{c, e f f}}
$$

where $\sigma_{c, e f f}$ represent the effective loss constituent of the microstrip conductor. Equating Eqn. (29) and (30), this effective conductivity $\left(\sigma_{c, \text { eff }}\right)$ of the microstrip can be deduced as:

$$
\sigma_{c, e f f}=\frac{\varepsilon_{e f f}(0, u) \exp \left[-2.4\left(Z_{0}(0) / \eta_{0}\right)^{0.7}\right]}{Z_{0}^{2}(0) w^{2} \sigma_{c}}
$$

Incorporating Eqn. (31) into the Cole-Cole expression, an imaginary part of the effective permittivity of the $\operatorname{microstrip}\left(-j \varepsilon_{c}^{\prime \prime}(\omega)\right)$ can be written as:

$$
-j \varepsilon_{c}^{\prime \prime}(\omega)=-j \frac{\varepsilon_{e f f}(0, u) \exp \left[-2.4\left(Z_{0}(0) / \eta_{0}\right)^{0.7}\right]}{\omega \varepsilon_{0} \varepsilon_{u}^{\prime}(\omega) Z_{0}^{2}(0) w^{2} \sigma_{c}}
$$

Lastly, the substrate loss $\left(-j \varepsilon_{d}^{\prime \prime}(\omega)\right)$ can be specified as $-j \varepsilon_{d}^{\prime \prime}(\omega)=-j \sigma_{d} / \omega \varepsilon_{0} \varepsilon_{r}$ where $\sigma_{\mathrm{d}}$ is the conductivity of the lossy substrate. Hence, the resultant equivalent Cole-Cole diagram representation of the microstrip system can be written as,

$$
\varepsilon^{*}(\omega)=\varepsilon_{u}^{\prime}(\omega)-j \varepsilon_{u}^{\prime \prime}(\omega)-j \varepsilon_{c}^{\prime \prime}(\omega)-j \varepsilon_{d}^{\prime \prime}(\omega)
$$

\section{Simulations AND RESUlts}

\section{A. Lossless Case}

Two dielectric substrate are chosen for this study which are $\varepsilon_{r}=10.5$, and, $\varepsilon_{r}=12.5$, and $\varepsilon_{r}=8.0$. The line-width to substrate thickness ratio $(w / \lambda)(h / \lambda)$ is taken as 1.0, 2.0, 4.0, and, 6.0 .

Considering the equivalent Cole-Cole diagram of the testmicrostrip depicted in Fig. 6, the simulation results reveal that the non-fringing part of the reactive energy in the microstrip reduces when the $(w / \lambda)(h / \lambda)$ ratio increases. For example, about $10 \%$ reduction of this non-fringing energy is observed when the $(w / \lambda)(h / \lambda)$ ratio is increased from 1.0 to 2.0 for both dielectric materials. The results indicate that the extent of the energy is confined within microstrip structure without proliferation since fringe fields increases with the dielectric constant of the substrate.

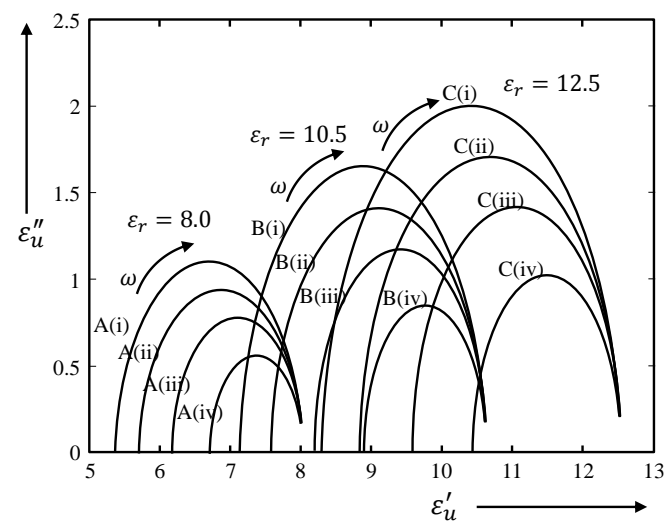

Fig. 6. Cole-Cole diagram for the test microstrip structures. CurvesA $: \varepsilon_{\mathrm{r}}=$ 8.0; Curves B $: \varepsilon_{r}=10.5$; Curves C $: \varepsilon_{r}=12.5$; (i) $(w / \lambda)(h / \lambda)=1.0$ (ii) $(w / \lambda)(h / \lambda)=2.0$; (iii) $(w / \lambda)(h / \lambda)=4.0$ (iv) $(w / \lambda)(h / \lambda)=6.0$.

\section{B. Lossy Case}

The dielectric substrates considered in this study are $\varepsilon_{\mathrm{r}}=$ $10.5, \varepsilon_{r}=12.5$, and, $\varepsilon_{r}=8.0$. The conductivity of the copper strip is $5.76 \times 10^{7} \mathrm{~S} / \mathrm{m}$. The line-width to substrate thickness ratio $(w / \lambda)(h / \lambda)$ is taken as 1.0 and 2.0.

Considering the equivalent Cole-Cole diagram of the testmicrostrip depicted in Fig. 7, the simulation results reveal that the non-fringing part of the reactive energy in the microstrip reduces as the $(w / \lambda)(h / \lambda)$ ratio increases. For example, about $8 \%$ reduction of this nonfringing energy is observed when the $(w / \lambda)(h / \lambda)$ ratio is increased from 1.0 to 
2.0 for both dielectric substrate materials considered. As expected, the results also indicate that the extent of the energy confined within microstrip structure (without proliferating as fringe fields) increases with the dielectric constant of the substrate; and, the reduction of energy can be observed when the substrate and conductor losses are included in the calculation.

Generally, the Cole-Cole arc is symmetrical about a line through the center, parallel to the $\varepsilon^{\prime \prime}$ axis. But those obtained via simulation results (Fig. 7) do not have this symmetry. The plots showed skewed arcs. This skewed representation in material-based Cole-Cole diagram is known as the Davison-Cole diagram [9]. Skewed arcs in Fig. 7 (microstrip-based Cole-Cole diagram) are due to the addition of the terms representing dielectric and conductor losses in Eqn. (33).

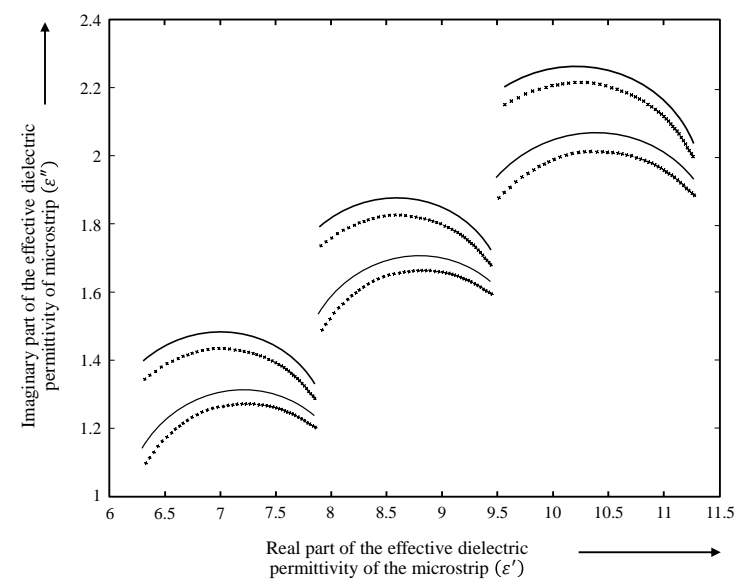

Fig. 7. Cole-Cole diagrams of test microstrip structures. $\sigma_{\mathrm{d}}=0.5 \mathrm{~S} / \mathrm{m}$. Curves A $: \varepsilon_{r}=8.0$; Curves $\mathrm{B}: \varepsilon_{r}=10.5$; Curves $\mathrm{C}: \varepsilon_{r}=12.5$; (i) $u=(w / \lambda)(h / \lambda)=1.0$; (ii) $u=(w / \lambda)(h / \lambda)=2.0 ; \mathrm{f}_{\mathrm{A}}: 20-60 \mathrm{GHz}$ and $\mathrm{f}_{\mathrm{B}}$ : 20-80 GHz. — Lossless, xxxxxx Lossy.

\section{CONCLUDING REMARKS}

Using the dielectric relaxation concept, the microstrip-based Cole-Cole diagram is derived and used to represent the microstrip structure performance. The proposed method offers a new and computationally tractable approach (via the Cole-Cole diagram concept) to study the frequency-dependent aspects of microstrip structure. It is a useful strategy towards ultra-high-frequency circuit designs.

\section{REFERENCES}

[1] E. Yamashita, K. Atsuki, and T. Veda, "An accurate dispersion formula of microstrip line for computer-aided design of microwave integrated circuit," IEEE Trans. Microwave Theory Tech., vol. MTT-27, pp. 1036-1038, Dec. 1979.
[2] A. K. Verma and R. Kumar, "A new dispersion model for microstrip line," IEEE Trans. Microwave Theory Tech., vol. MTT-46, pp. 1183-1187, Aug. 1998.

[3] E. Riande and E. Saiz, Dipole Moments and birefringence of Polymers, New Jersey: Prentice Hall, 1992.

[4] G. R. Bhartia, P. Bahl, and I. Ittipiboon, A. Microstrip Antenna Design Handbook, Artech House, Inc, 2001.

[5] E. Hammerstad and O. Jensen, "Accurate models for microstrip computer aided design," IEEE MTT-S Int. Microwave Symp. Dig., New York, NY, June 1980, pp. 407-409.

[6] K. C. Gupta et al., "Microstrip Lines and Slotlines," Dedham, MA: Artech House, 1996.

[7] M. Kirschning and R. H. Jansen, "Accurate model for effective dielectric constant with validity up to millimeter-wave frequency," Electron. Lett., vol. 18, pp. 272-273, Jan. 1982.

[8] V. V. Daniel, Dielectric Relaxation, London: Academic Press, 1967.

[9] P. S. Neelakanta, Handbook of Electromagnetic Materials: Monolithic and Composite Versions and Their Applications, Boca Raton, FL: CRC Press, 1995.

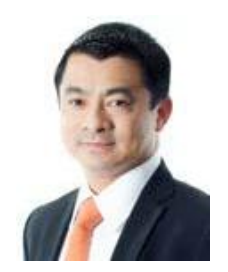

Settapong Malisuwan was born on $24^{\text {th }}$ March 1966 in Bangkok, Thailand. He received his $\mathrm{PhD}$ in electrical engineering (telecommunications), specializing in mobile communication systems from Florida Atlantic University (State University System of Florida), Boca Raton in 2000. He received an MSc in electrical engineering in mobile communications system, from George Washington University in 1996, an MSc in electrical engineering in telecommunication engineering from Georgia Institute of Technology in 1992 and a BSc in electrical engineering from the Chulachomklao Royal Military Academy, Nakhon-Nayok, Thailand in 1990. He served in the Royal Thai Armed Forces for more than 25 years and is currently the Vice Chairman of National Broadcasting and Telecommunications, Bangkok, Thailand. His research interests are in efficient spectrum management and Telecommunications policy and management in Thailand. Col. Dr. SettapongMalisuwan is currently the Elected Vice Chairman and Board Member in the National Broadcasting and Telecommunications Commission, Thailand.

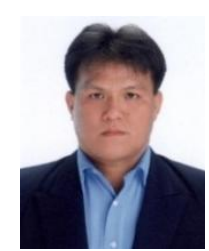

Jesada Sivaraks was born on $12^{\text {th }}$ May 1970 in Bangkok, Thailand. He received his MSEE degree from Oklahoma State University in 1996 and BEng from King Mongkut"s Institute of Technology, Thailand. He completed his $\mathrm{PhD}$ in electrical engineering at Florida Atlantic University, Boca Raton, FL in 2001. Since 2011, he has been working in National Broadcasting and Telecommunications Commission as the Secretary to the Vice Chairman. His PhD work is on the system aspects of Bluetooth, WLAN and Mobile IP/CDPD. His current research interests are in telecommunication planning and related system analysis and efficient spectrum management. He is a member of Tau Beta Pi, Florida Epsilon and was an Honorary Advisory's Chairman of Science \& Technology committee of Parliament in 2009

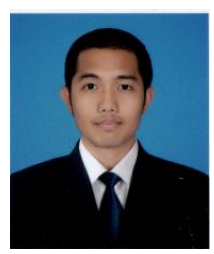

Nattakit Suriyakrai was born in Khonkhaen, Thailand on $22^{\text {nd }}$ March, 1987. He received his Bachelor of Liberal Arts in Japanese Language from Thammasat University in 2010. He has been working as an Assistant to Vice Chairman in National Broadcasting and Telecommunications, Bangkok, Thailand since November 2012. His research interests management. are in technology management and spectrum 